\title{
Quantum Gravity Theory Founded on Device That Generates Energy from Relative Acceleration Amongst Charged Particles Electrostatically Interacting Under Curvature Deviation
}

\author{
Samuel P. Moss II \\ Moog Inc. Aerospace and Defense Company, 400 Jamison Road, Elma, NY 14059 \\ E-mail: smoss3@moog.com
}

\begin{abstract}
This is an introduction to a new concept of quantum gravity that seamlessly merges General Relativity to the Standard Model. Based upon a novel patent-pending magnetic confinement method that was designed to emulate how our sun confines and rotates charged particles about a singularity; this confinement method uses a collective of off-centered confinement coils that are directed to curve rotating charged particles about a singularity in a way that allows charged particles to relatively accelerate from geodesic deviation. With this confinement method the subtle Relative Accelerated Energy (RAE) from deviating charged particles has the capability to be focused and exponentially increased relative to the mass energy of a closed system; which allows for a simple pathway to understand how black holes operate at their singularities. While in the pursuit of proving that this novel method of confinement mimics how our sun operates; I was also able to develop a logical explanation of how our sun reverses its magnetic poles and cycles using the core principles of Michael Faraday. If this concept of quantum gravity is correct, there is a simple explanation for the additional observed gravitational force about the galaxies that are said to obtain dark matter. In short, this theory of quantum gravity has the potential to fully discredit the existence of theorized dark matter with a simple experiment.
\end{abstract}

Key words: quantum gravity, general relativity, acceleration, geodesic deviation, Riemann Curvature

\section{Introduction}

Originally, I developed the Nuclear Electromagnetic Shaping Accelerator Reactor (NESAR) [1] to be a logical approach to sustainable fusion by designing the first reactor that truly confines similarly to how our sun operates. In an effort to express the validity of this postulation, I attempted to supplement a logical method that would explain how the sun reverses its magnetic poles based upon how the NESAR confines charged particles. Astonishingly, I was able to develop a straightforward explanation on how the sun's core perpetuates, which then bolstered me to further explore possible effects upon the charged particles within the confines of the NESAR. After months of closely analyzing the possible effects this confinement could have on interacting charged particles, I realized these charged particles have the capability to deviate in their curvatures from the Single Relative Center Point (SRCP). This deviation is due to Converging Geodesic Deviation (CGD) [2] , which causes subtle relative acceleration amongst electrostatically interacting charged particles that we may be observing as gravity.

Possibly the most significant capability of the NESAR is its aptitude to focus the combined electrostatic force of confined electrons to a singularity at the SRCP; resulting in a maximized negative well potential for ions to accelerate towards for improved initial fusion rates. So, upon understanding that subtle RAE due to geodesic deviation could be the cause for gravity, I explored the possibility of exponentially compounding this possible energy for utilization. This approach to understanding gravity 
allows for the generated energy of a black hole to finally be calculated at the singularity, while creating a way to supersede the baryonic limitations of energy that are observed and categorized as dark matter.

In this paper, I will initially review the design of the NESAR. To add validity to this method of confinement, I will review a novel law-based theory that logically applies Faraday's law of induction to explain how the sun perpetuates. Lastly, I will present this novel approach to understanding gravity at the quantum level. The concepts and theories based on the NESAR are solely based upon the proven mathematics and laws of physics, so there are no exceedingly complicated postulations to understand or to build upon.

This will be the first of two papers in reviewing the capabilities of the NESAR. The second paper will focus on the improved fusion capabilities of the NESAR in comparison to the Tokamak and other cusp confining reactors without the assistance of gravity. Even though this concept was originally developed as a fusion reactor; I am initially presenting the quantum gravitational theory tied to this device in an effort to reach a broader audience who is more familiar with general and special relativity instead of the small minority that purely focuses on fusion. In addition, most physicists feel that sustainable fusion isn't feasible without the assistance of the gravitational force in the first place. So, if my concept of quantum gravity seems logical, then reviewing the details of the fusion capabilities is a moot point for most physicists.

\section{The Logic in Designing A Reactor that is Supposed to Confine Like the Sun}

The surface of the sun is full of plasma, which is one of the four states of matter which is formed at high temperatures and consists of freely moving protons, positively charged, and free electrons, negatively charged. Besides being able to observe its bright surface called the photosphere; probably the other most observable feature to the sun are its sunspots that form on its surface every 11 years when the sun is at its most active. These spots have a much stronger magnetic field strength than the other surface areas of the sun. In addition, these spots are also at a cooler temperature in comparison to the surrounding areas. Due to these spots being concentrations of magnetic strength, these are the areas that will have the greatest observable influences on the plasma, charged particles. Plasma can be manipulated by magnets and will travel in the direction of the magnetic fields.

No one knows if sunspots are formed from inadequacies to the sun's confinement system, but the main thing to take away is that they are for the most part circular in shape and are gigantic magnetic fields that traverse through photosphere. In observing most sunspots, they look as if a collective of charged particles are being pulled into the confines of the sun. For this to happen, a good portion of these sunspots would have to be a gargantuan clockwise moving current; which results in creating the inward-directed magnetic field. If you are not familiar with electromagnets, the right-hand rule is used to determine the direction of the magnetic fields created in electromagnets and coils. The way this rule works is by making a big thumbs up with your right hand and have the curled finger go in the same direction as the current. When you do this, the thumb will be the direction of the magnetic field created. In the next picture below one can see the plasma from the photosphere being stretched and pulled into the sun's core. This same magnetic effect would occur if one were to place a clockwise current through a coil of wire. 

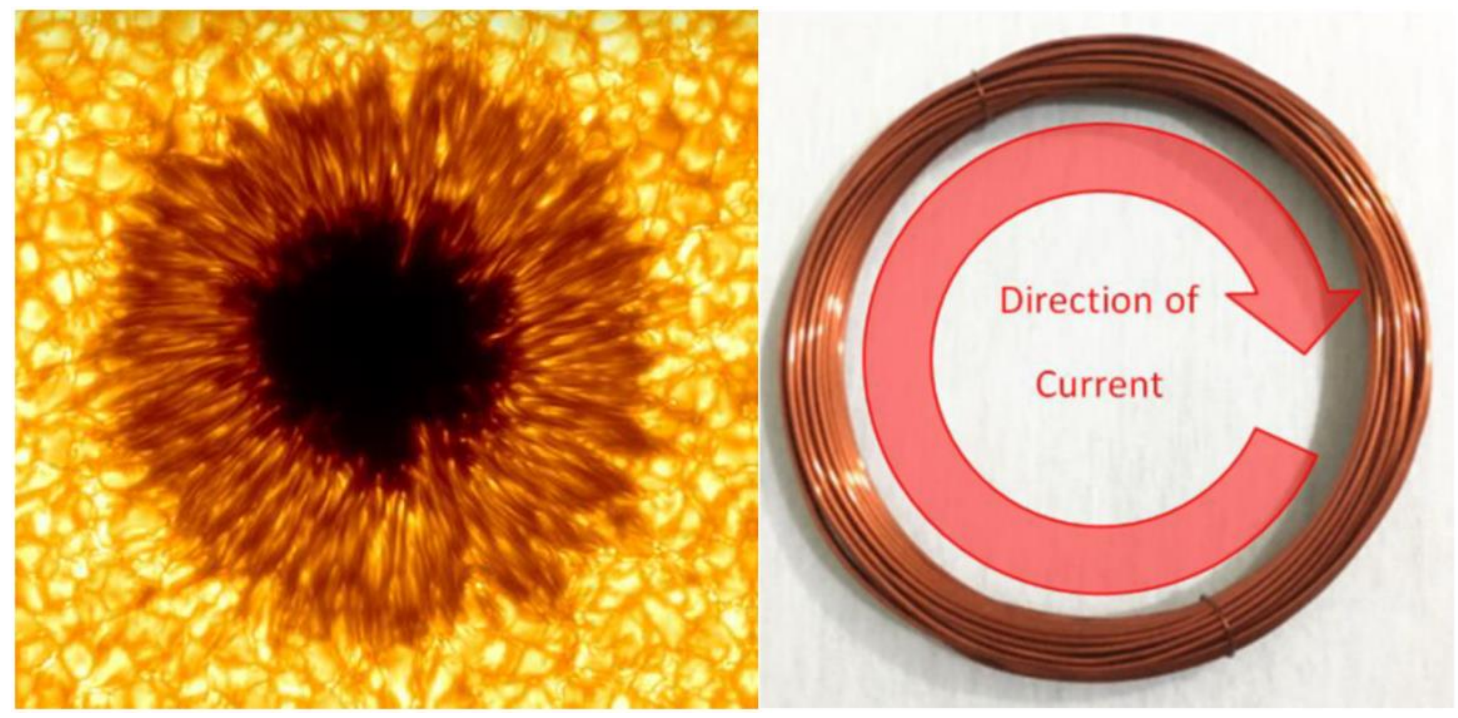

Our sun is not the most efficient star at fusion, which is a good thing. If the sun was too efficient at perpetuating fusion, it would burn out much faster. Sunspots are a reoccurring phenomenon in the sun's confinement system; but one of the most important things that can be assumed from the observation of sunspots is that there is a great probability that there is a current-carrying layer under the photosphere made of thousands to possibly millions of these weaker and less dynamic contiguous clockwise currents. This realization initialized me to design a spherical shaped magnetic confining apparatus that consisted of numerous toroidal magnetic coils that would have an inward directed magnetic field.

In designing the confinement apparatus, I used a multisided pentacoil design to evenly disperse the toroidal magnetic coils as the surface of a sphere. This pentacoil design for the confinement apparatus is not an essential feature. Below you can see the pentacoil design and how it collectively makes up the spherical confinement apparatus for fusion.
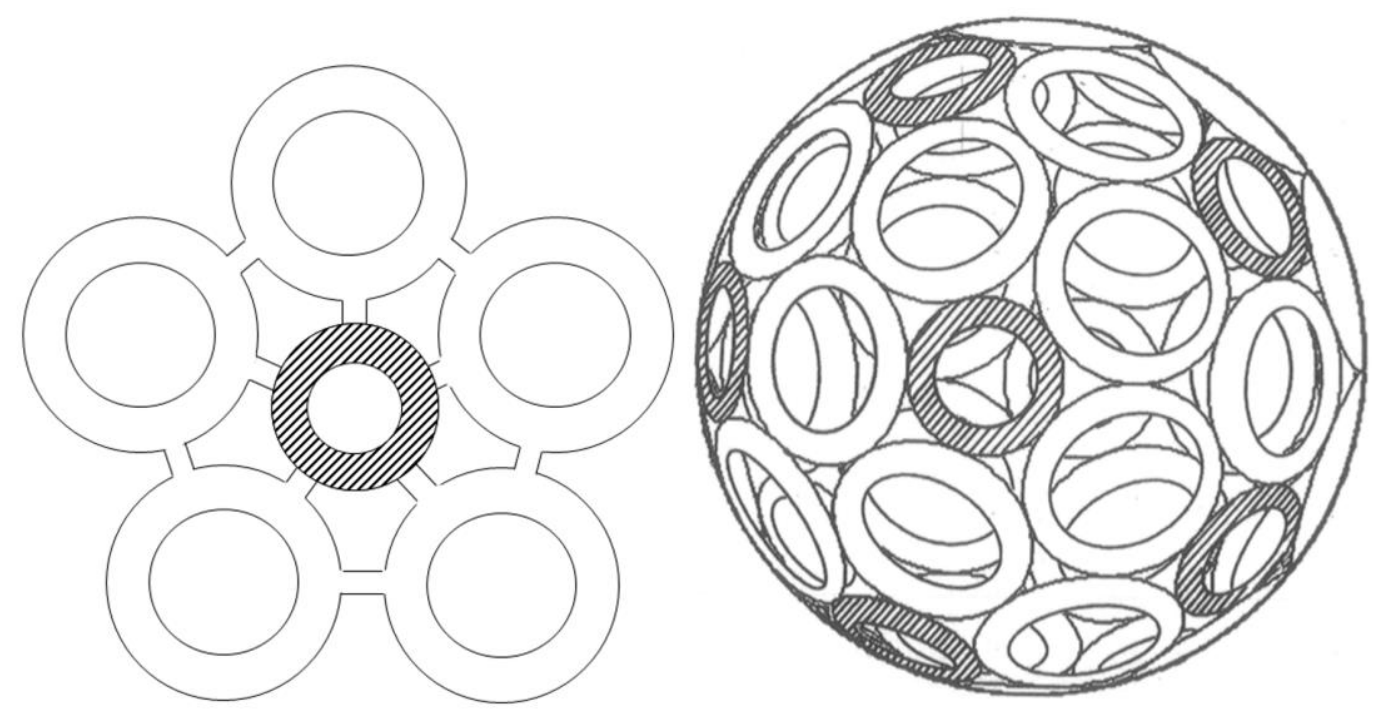

When looking at the spherical confinement apparatus that is supposed to operate within a vacuum chamber; one can falsely assume that the NESAR is supposed to simply confine charged 
particles to the center of the apparatus. If this were so, the most import feature of the NESAR is that every confinement coil, besides the vertical axis, is directed off center to promote a collective directional rotation to the confined charged particles within the confinement apparatus. Angling the magnetic confinement fields to spherically rotate charged particles; allows the NESAR to quickly perform what takes the sun thousands of years to do naturally through sheer size and induction from an increasing well potential at the center of its confinement. The natural evolution of the sun's rotating core will be covered later on in the paper. Being able to spherically rotate charged particles is vital to the Sun and the NESAR in having the capability to effectively confine and perpetuate without catastrophic magnetic reconnections that could disrupt the confinement. Depicted below is a cross-section top view of how the confinement coils are collectively angled to promote a rotation to the confined charged particles.

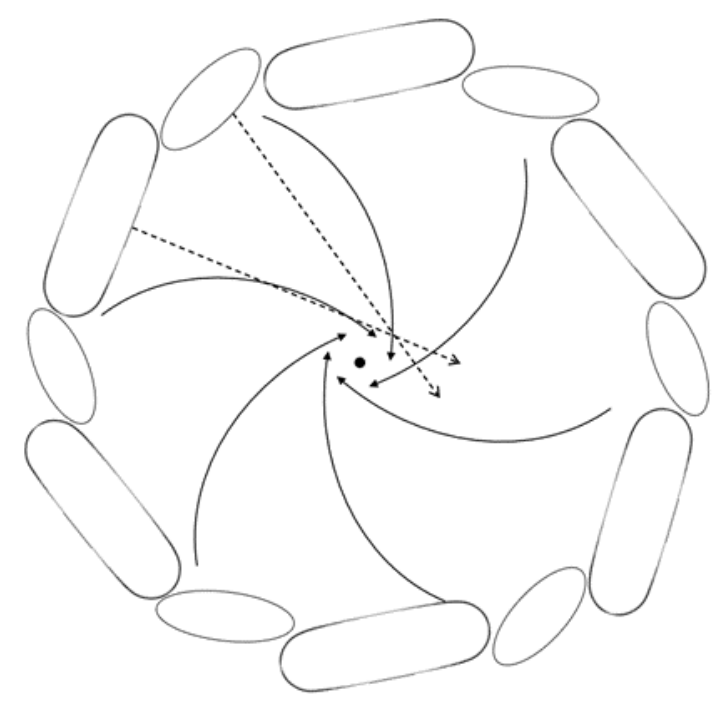

To better show how the magnetic fields of the NESAR will confine and rotate charged particles within the confinement apparatus, the following depiction has two 3D diagrams showing the crosssection of the confinement apparatus to highlight the angling of the toroidal magnetic coils. The diagram on the left is a cross-section view from a top looking down on the NESAR confinement apparatus. From this diagram, one can observe the toroidal magnetic coils that are slightly directed off center to push a rotational pattern upon the confined charged particles. The angled toroidal magnetic coil fields on this plane are depicted in blue. The diagram on the right is a cross-section view from an upright perspective of NESAR confinement apparatus. In the diagram on the right, the angled toroidal magnetic coil fields directed to the background are green; while the toroidal magnetic coil fields directed to the foreground are blue. In this same diagram, the toroidal magnetic coil fields on the vertical axis directed to the center of the confinement apparatus are orange. 

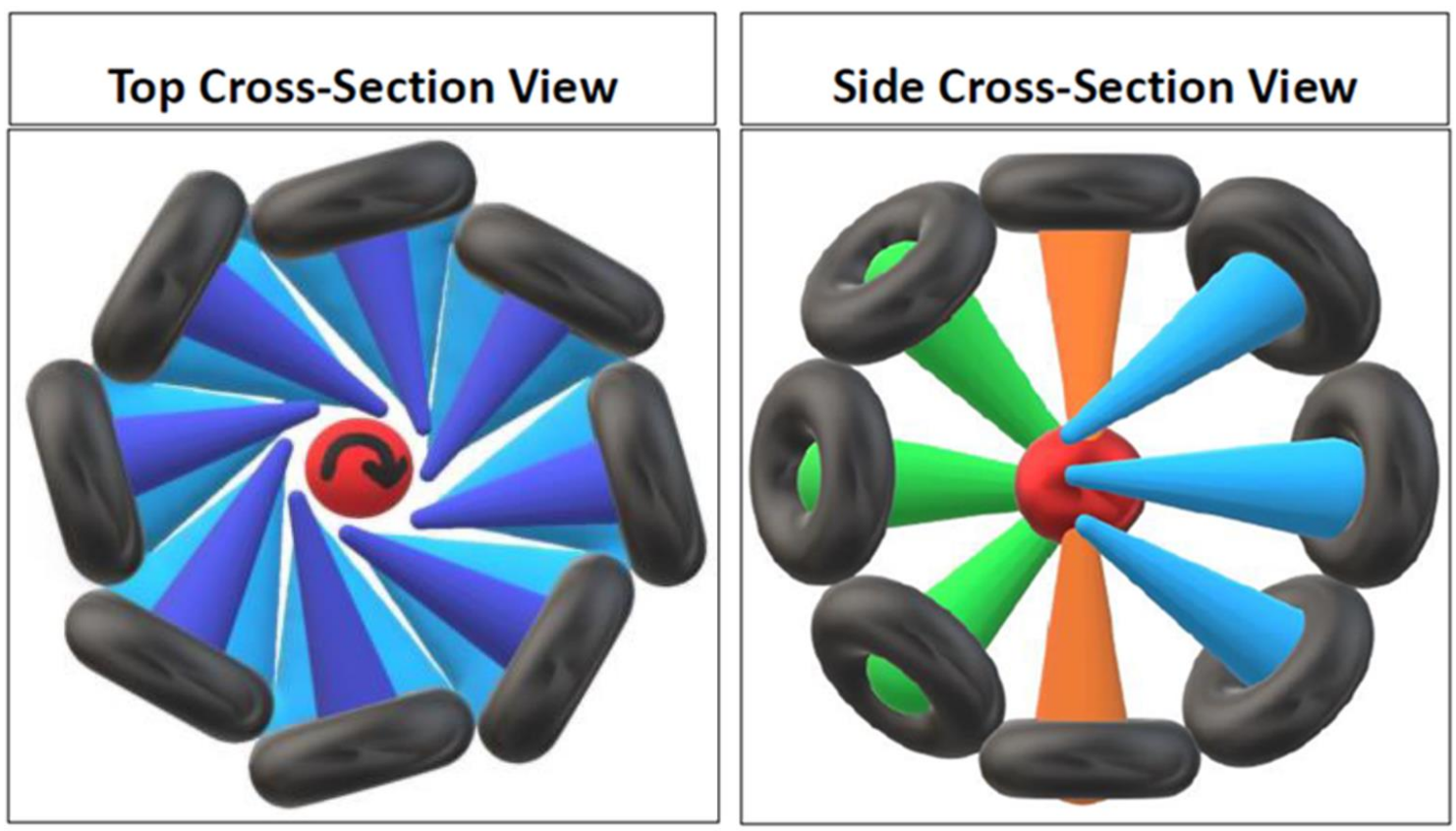

Magnetic fields will not cross each other, they merely push against one another. This means that the angled toroidal magnetic fields will layer upon each other while rotating confined charged particles spherically. This increases the confinement's capability in trapping charged particles while at the same time driving charged particles to rotate collectively to create a dominate magnetic field within the NESAR confinement apparatus. In remembering that an electromagnet is only charged particles moving as a collective in a rotational direction, the confined particles within the NESAR will collectively exhibit the same electromagnetic properties as charged particles flowing through a coil. Thus, creating an observable primary magnetic field for the system just like the sun.

\section{The Approach to Perpetuating Fusion Like the Sun}

The NESAR is the only magnetic cusp confinement device that collectively rotates confined particles spherically. Magnetic cusp confinement reactors attempt to use Inertial Electrostatic Confinement (IEC) as well to accelerate charged particles for fusion reactions. The sequence of this type of fusion is by initially magnetically confining enough electrons to create a negative well potential. Once a negative well potential is established, protons of a hydrogen isotope are then injected within the confines of the confinement apparatus to be accelerated towards the negative well potential to fuse.

Previous developers of magnetic cusp confinement methods have been unsuccessful at producing fusion rates high enough to be effective for numerous reasons that I will cover later on in the second paper, but the one thing that they are right about is using the initial confinement of electrons to establish a negative well potential for perpetuating fusion. The reason that using magnetic confinement to produce a negative well potential may be the most effective approach to accelerating protons for fusion, is because there is a great probability that our sun and most stars initially confined in this manner.

After the Big Bang, the universe was a hot soup of neutrons, protons, and electrons. As star confinements, which are similar to the NESAR without the angled fields, confined these particles; the least massive electrons were the easiest and first to be spherically rotated and confined. Initially 
confining the electrons in this manner, creates the rotating negative well potential that is needed to accelerate and recirculate the surrounding protons for perpetuating fusion.

In the same manner as the sun and other stars, the NESAR initially confines by establishing a spherical rotating collective of electrons, which are initially injected within the confines of the apparatus; the confined electrons electrostatically interact at the Single Relative Center Point (SRCP) to create a closed system with a collective maximum negative well potential singularity. Upon establishing this negative well singularity, positively charged ions for fusion are able to be electrostatically accelerated towards and circulated about the SRCP for maximized efficiency in fusion collisions. The process of establishing a well potential in stars would not be as controlled and sequenced as it would be in a NESAR confinement, because the less massive electrons have the advantage in establishing a negative well potential prior to a possible positive well from the confinement of protons.

Below is an top view cross-section image depicting this interaction. On the left, a magnified view of the confinement of spherically rotating electrons is shown to electrostatically interact to create a maximized negative well potential singularity at the SRCP. In the top view cross-section depicted below on the right, injected protons are shown being accelerated towards the SRCP.

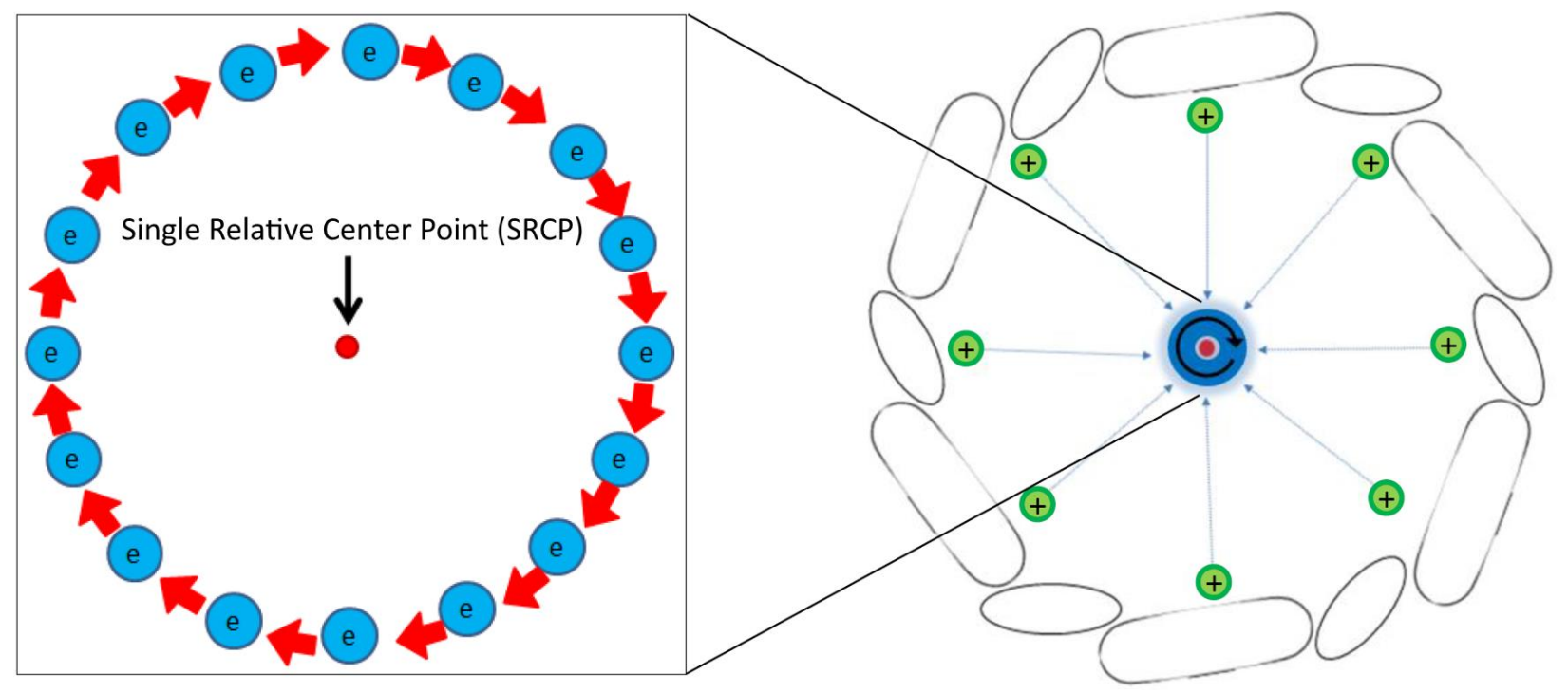

Since a created singularity is the focus point for proton collisions for fusion, the fusion rate surpasses all previous Magnetic Confining Inertial Electrostatic Confinement (MCIEC) concepts. The NESAR is the first MCIEC to spherically rotate its confinements, which means positive ions that are not initially fused are able to be re-circulated within the confines of the NESAR to allow protons the opportunity to fuse again. These improvement factors and more will be covered later on in the second paper to explain how the NESAR produces higher sustainable fusion rates at a much smaller confinement size when compared to the current leading methods toward sustainable fusion.

To clarify, I am not postulating that the sun's core is designed of confining coils like the NESAR. On the contrary, I believe that the heart of the core of the sun and most stars consist of rotating charged particles. The NESAR is just a means of simulating this rotation of charged particles at the cores of stars. It is common knowledge that our sun is about $90 \%$ hydrogen and $10 \%$ helium; but by the end of this paper is will be fully understood how a core that is slightly less than $1 \%$ of volume of the sun is able to 
hold more than a $1 / 3$ of the sun's mass, reverse its magnetic field, and generate the RAE we know as gravity.

\section{Proving that the NESAR Confines Like the Sun By Explaining How the Sun Reverses Its Magnetic Field}

The NESAR was developed in an attempt to reverse engineer our own sun. So, the collective flow of charged particles within the NESAR confines should provide some type of understanding in how the sun operates. In my research of the sun, I realized that no other physicist or cosmologist who studies helioseismology has come up with a logical explanation of how the sun reverses its magnetic poles while progressing through its solar cycles. Understanding this quandary, I knew if I were able to logically explain this phenomenon it would add validity to the NESAR confinement method.

There are many layers to the sun that have a multitude of different features. Since I am trying to focus on magnetic pole reversals, it must be coming from one source and that source should be the sun's core. The sun is a continually changing environment, so it should be assumed that its core is doing the same. If this is true, then induced effects of the core should be very observable and constantly altering as well. Maybe it's because I am a huge fan of the self-taught English scientist Michael Faraday and the eccentric engineer Nikola Tesla; that I chose to approach better understanding how our sun operates in merging the general concepts of these two legends.

In studying Faraday's experiments [3] , I remembered that the direction of inductance is not dependent upon the physical direction of the magnetic field of the source but is depended upon the change in magnitude of the magnetic field in a specific direction. So, applying this concept to how my NESAR confined, the system's magnetic field is generated from the confinement of charged rotating particles. Unlike the moving bar magnet used in Faraday's experiment as the magnetic field source, the only way that the magnetic flux could change in the NESAR confinement or the sun's core is by altering the rotational speeds of the confined charged particles. To get a better idea of this concept, look at the depiction below. In the diagram on the left, the created magnetic field will be directed downward. As the rotational speeds of the confined charged particles increase; the more charged particles will pass through the imaginary blue plane over time. This increase in rotational speed results in the NESAR or sun's core to increase in current, causing the system as a collective to increase in voltage.
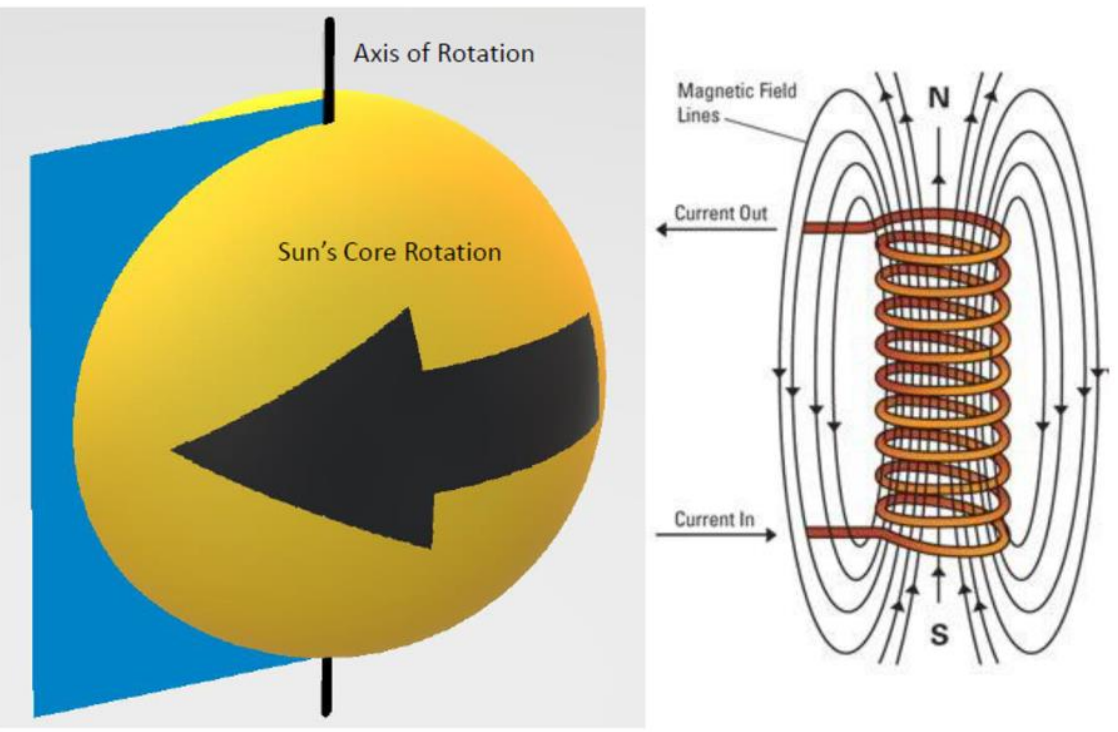
Since the NESAR and the sun are rotating balls of charged particles, plasma; it was logical to relate how the induced current generated within tokamaks may be similar to how it may occur within the sun's core. Even though tokamaks are extremely unsuccessful due to magnetic reconnection failure; experts know how the reconnection occurs. The reconnection failure in tokamaks is call a sawtooth reconnection. This reconnection occurs when the opposing induced current's field from the main confinement of charged particles is pinched and severed by the field of the main current of confined charged particles. This happens because the tokamak's poloidal field currents run parallel to the current of confined charged particle. So, as a tokamak increases in its confinement of charged particles; the induced current's field gets pinched between the field of the main current of charged particles being pulled towards the fields of the poloidal currents that are affixed to the outside of the confinement apparatus. This pinching of the induced field, causes an explosive surge of kinetic and thermal energy that causes massive disruptions in the tokamak confinement. Below are a couple of diagrams that depict this sawtooth failure [4]. Please notice how the opposing induced current is created in diagram $A$, in yellow. In diagram $B$, the main current's field is being pulled towards the parallel running poloidal currents, pinching the induced current's field.

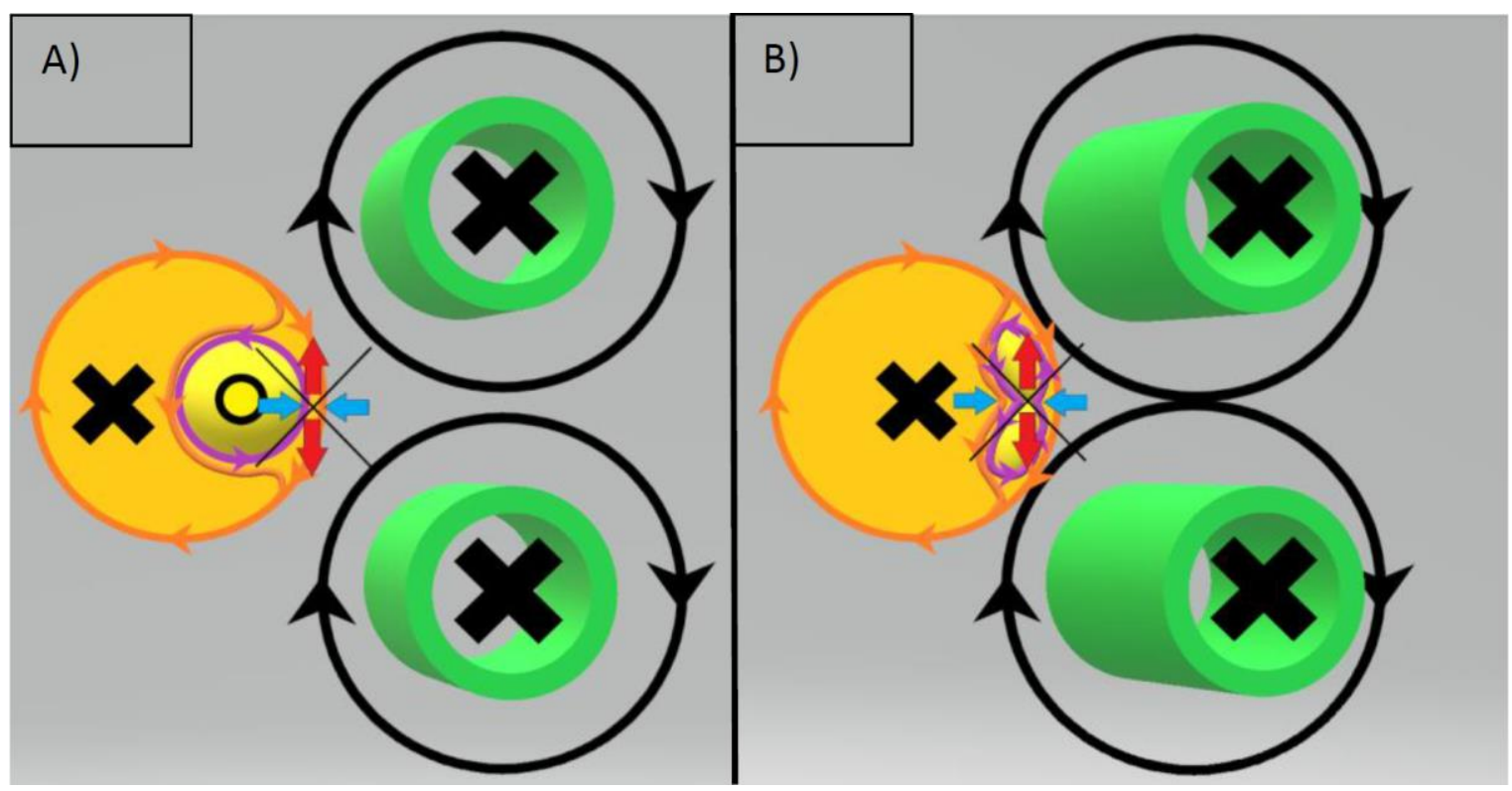

I will cover much more in dept how magnetic reconnection occurs in the second paper and explain how the NESAR eliminates this catastrophic failure issue. The main reason that I am bringing up the tokamak sawtooth failure at this time in the paper is to provide a depiction of the type of induced current that is more than likely occurring in the sun's plasma core. This induced current plays an extremely important role in the perpetuation and existence of our own sun.

Since the magnetic field of our sun's core is constantly changing then there must be the presence of inductance in the sun's surrounding environment. In Faraday's experiments, inductance occurs in the surrounding coils around the source of the changing magnetic flux. Thinking of his experiments made me wonder if the equipment that we are using to observe the sun's magnetic pole reversal are simply coils that are recording the sun's core change in magnetic flux instead of its actual magnetic field. 
Because of this uncertainty of what NASA is recording as the sun's magnetic field, I started to research the equipment that is used to record the sun's magnetic field. NASA uses Search Coil Magnetometers (SCM) [5] to observe the sun's magnetic field. SCMs are basically copper coils wound around a high magnetic permeability core. This magnetic core concentrates magnetic field lines - and the magnetic fluctuations they carry - inside the coils. The fluctuations induce currents and electric voltage drops inside the core that can be measured and recorded by the instrument's electronics circuits. In general, these coils are recording the inductance given off by the sun's core. Their SCMs not only observe one direction, they record the magnitude of the induced electromotive force (EMF) on the $X, Y$, and $Z$ axes. Below are pictures of the two types of search coil magnetometers used to observe the sun's magnetic field. In the pictures you can physically see the coils wounded to obtain three axes.

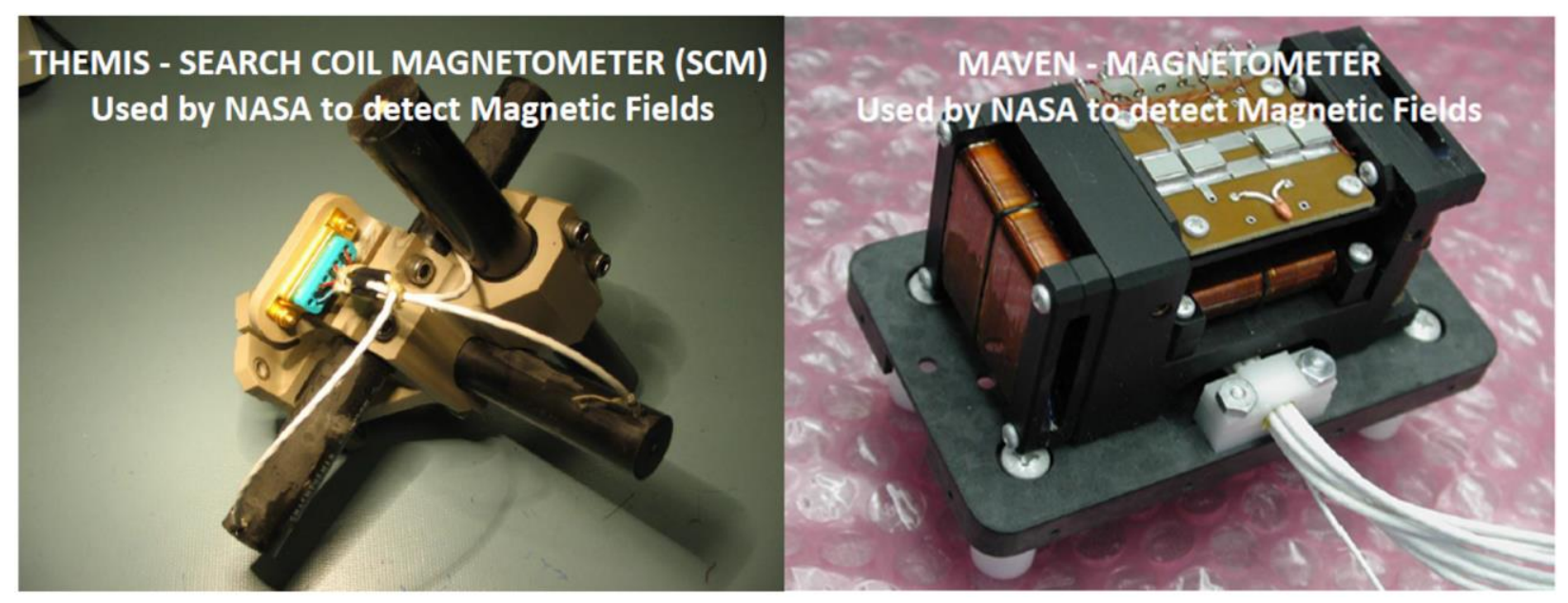

In researching NASA's SCMs, I was surprised that these recordings were not more publicly presented as recorded inductance readings instead of the actual magnetic field of the sun's core. Faraday discovered and clearly conveyed how inductance occurs in conducting coils almost over 200 years ago, I truly believe if the sun's pole reversal were presented as a shift in measured inductance, those who study helioseismology would have figured out much earlier that these sensors are not measuring actual flips in the magnetic field of the sun's core.

After researching the NESAR and NASAs SCMs in a Faradayan manner and realizing that the sun's core could obtain a same rotational direction of charged particles while presenting shifts in inductance; It was time to approach learning how the sun's magnetic pole reversals occur in a Teslan approach. So, if man is actually observing the sun's inductance, then the recordings that are being captured by NASA's SCMs are actually the sun's electromotive force (EMF).

In the study of electric motors, stators induce an EMF current upon the rotors to drive a rotation upon the motor's rotor. Any change in the magnetic environment of a coil of wire will cause a voltage (emf) to be "induced" in the rotor coil. A rotating confinement of charged particles would create a dipole magnet field similar to a coil. No matter how the change is produced, the EMF voltage will be generated. The change could be produced by changing the magnetic field strength, moving a magnet toward or away from the coil, moving the coil into or out of the magnetic field, or rotating the coil relative to the magnet. 
In the case of the sun's core or NESAR the constantly changing rotational speed is the variable that creates the EMF. To proceed further explaining this relationship I had to develop the below diagram to differentiate between the main current and the induced current.

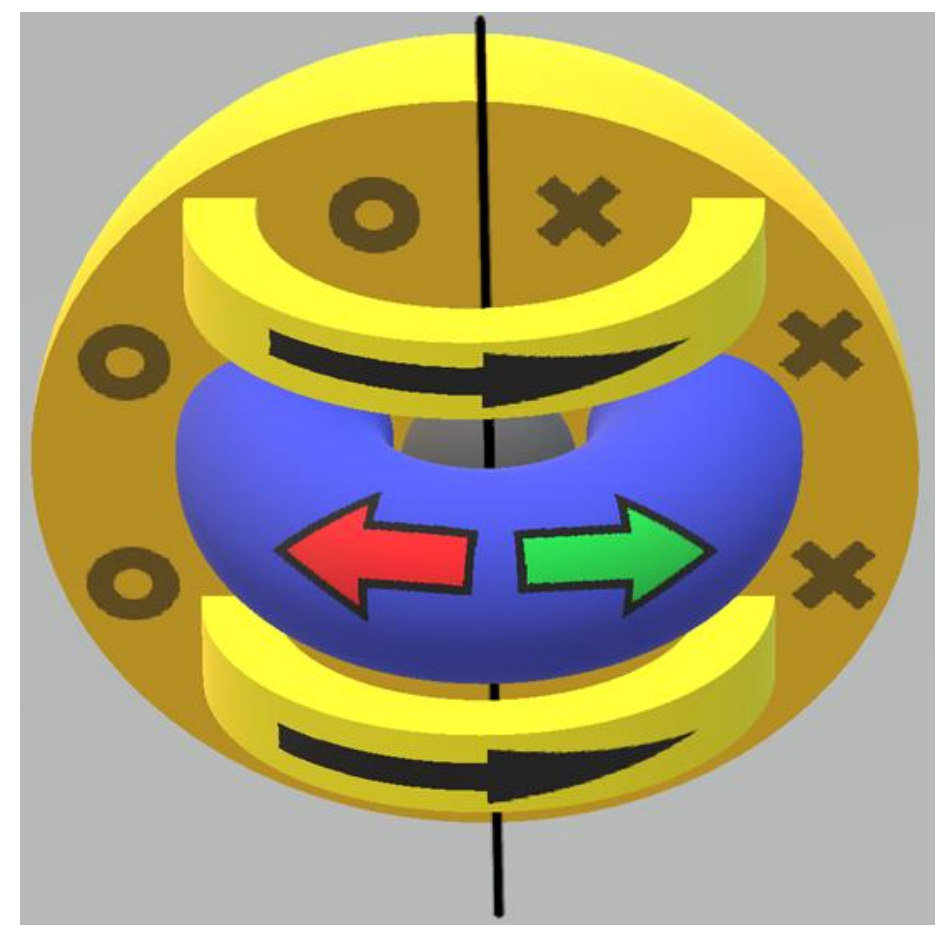

In the previous diagram, you can see the main current rotation of the sun's core is in yellow. The blue toroid is the induced current. There is a red and green arrow on this blue toroid to indicate that an induced current can either work to either increase or decrees the rotational speed of the system, as the system attempts to maintain its initial maximum rate of change where the induced current was at its greatest strength. In general, the sun's perpetuation is due to the law of conservation of energy; where the sun is attempting to maintain its maximum induced current by varying its rotational speed.

In order to graph the relationship between the main current and EMF; Faraday's fundamentals serve as a succinct summary of the ways a voltage (or emf) may be generated by a changing magnetic environment. The induced emf in a coil is equal to the negative of the rate of change of magnetic flux times the number of turns in the coil. Main thing to focus on for graphing this relationship for our sun's core is that the sun's EMF is the negative rate of change of the magnetic flux, which would be the negative rate of change of the systems rotational speed.

The following diagram and graph was developed to show how this relationship between rotational speed of the system is directly tied to its induced forces. On the X-Axis, is the EMF. The reason that EMF is on the X-Axis is because it is the negative rate of change of the rotational speed of the system that emits a magnetic flux in relation to its rotational speed. The EMF will shift from an aiding or opposing force towards the systems rotational speed. Again, this shifting in EMF is due to the system trying to maintain its greatest initial rate of change; but the most important thing to take notice is when the EMF is zero, is when we have been observing the sun's magnetic pole reversals with NASA SCMs. 
The following diagram is meant to be used in conjunction with the graph. It is meant to show how the induced current is supposed to increase or decrease the core rotation of the sun. Notice that the green indicates that the induced current is working to increase the rotational speed; while the red is opposing the direction of cores rotation, slowing the system's rotation. The EMF curve on the graph is color coated red and green as well to indicate when induced currents are working to increase or decrease the system's rotation. In the graph, notice that sun's rotational speed and magnetic flux is initially a dotted orange line. This is because logically there may be an instantaneous, not a gradual, maximum rate of magnetic flux that would have occurred when the system evolved to initially establish its rotation because the sun does not use angled confining magnets like the NESAR. If this is true, then there would not have been an established opposing induced current or EMF as well, which is why the corresponding EMF section of the graph is depicted with a dotted yellow line.
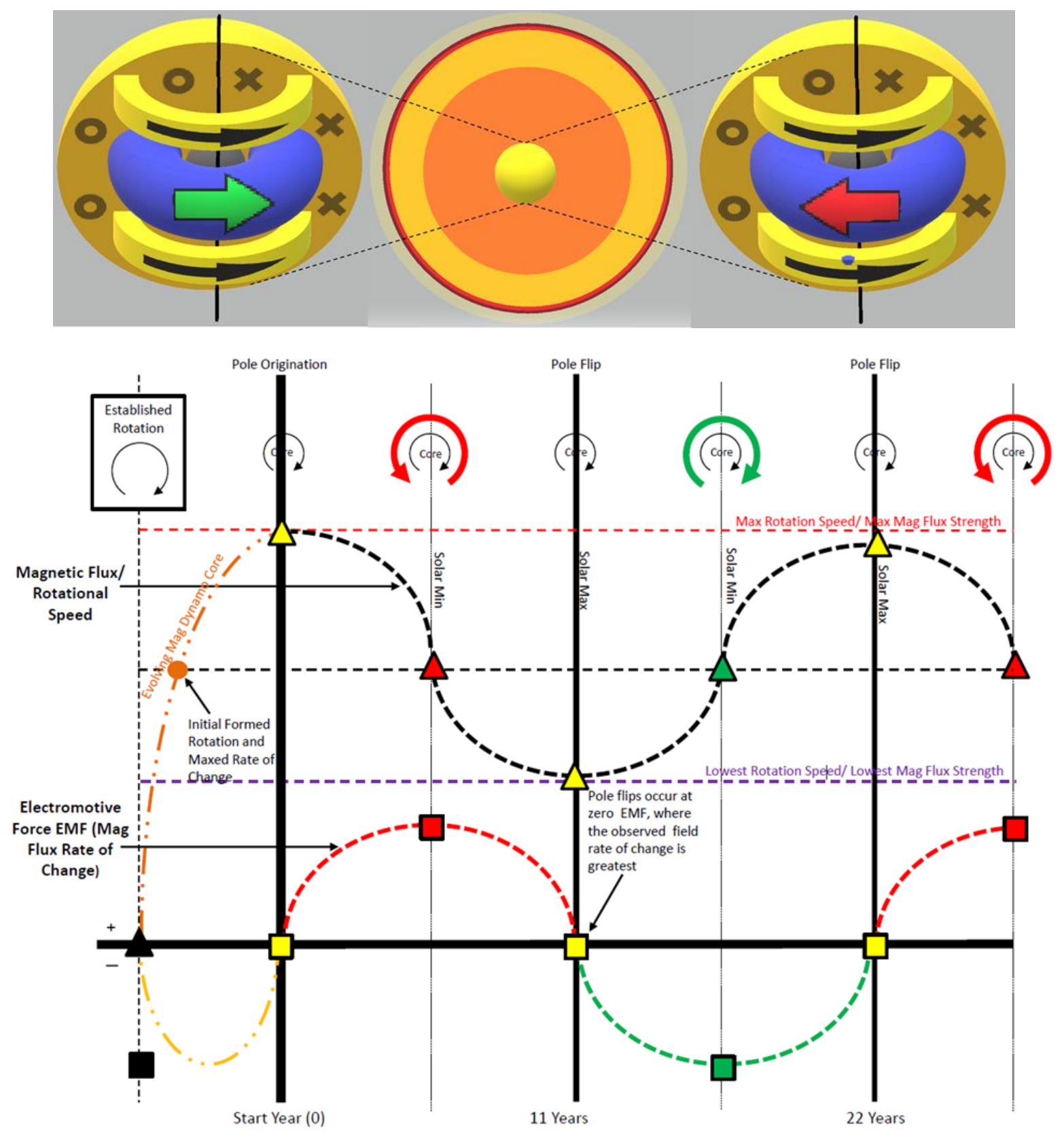
The main take-away from this graph, is to see how the induced currents within the sun's core act upon its rotational speed to create an alternating magnetic flux that produces the EMF that NASAs SCMs observe. This changing rotational speed is depicted by the black curve. Do recognize that the rotation never stops nor reverses, and that the magnetic pole reversals of the sun's core occurs when the rotational speeds are maximized and minimized on the curve.

One thing that I would like to clarify is that the EMF that NASAs SCMs are picking up is not the induced field created within the sun's core, even though this field congruently shifts with the sun's emitted EMF. The recorded EMF from the sun's core is merely the change in the rotational speeds of the sun's core that also congruently causes its magnetic flux to vary as well. This varying magnetic flux is what NASA's SCMs are picking up as reversing magnetic fields.

I believe that the Earth's core may operate very similarly to the sun's; just much less active. If it does, the reason why the Earth's poles change location is due to its core varying in rotational speed. As Earth's core slows in rotation, like a top, it starts to wobble; but once an induced current works towards aiding its rotational speed it should become more stable. Most important thing to understand is that the Earth's core will never physically rotate or flip, so any equipment dependent upon Earth's magnetic fields should only need minor calibrations to account for the shift in Earth's inductance when the time comes for Earth's magnetic field to reverse.

All things considered; this is a straightforward, no frills, theory of how our sun perpetuates. Not only is it logical, but it is also simple. It is a law-based explanation that incorporates Faraday's law with already recorded induced occurrences that scientists have documented in rotating plasmas when observing tokamaks. Most importantly this theory provides clarity on what NASA's SCMs are actually recording from the sun's core. After researching countless theories on the sun's magnetic pole reversals, this explanation makes the most sense with the current knowledge that we have on the sun's core.

\section{A Quantum Theory of Gravity Based Upon the NESAR}

When I initially developed the NESAR method of confinement, I was solely focusing on developing a more logical approach towards sustainable fusion by creating a fusion reactor that operated similarly to our own sun. Originally, I never conceived that this concept could ever be more than a possible method of fusion. It was not until I was able to develop a simple and logical theory on how our sun perpetuates and reverses its magnetic fields did it dawn on me to develop this concept further. The main reason I chose to explore the possibility that this type of confinement may be tied to generating gravity is because it is the only opposing argument scholars could make against my device being a fusion device that truly functions similarly to the sun. Before you start reading this section, I must inform you that I do use the terms gravity and RAE interchangeably.

Einstein maintained the philosophy that gravity isn't a force at all. He described it as a curvature of time and space caused by mass and energy. Most of his life he tried to form a rudimentary theory of gravitation that would unify the gravitational and electromagnetic forces together. For the first time there is a logical and testable quantum theory of gravity that unifies gravity to electromagnetism in a way that fully embodies what Einstein tried to achieve so many years ago.

The following gravitational theory proposes that charged particles collectively rotating with curved trajectories about a Single Relative Center Point (SRCP) while interacting electrostatically under 
the effects of curved trajectories will undergo relative accelerated interactions from curvature deviation also known as Converging Geodesic Deviation (CGD). This energy from relative accelerations is simply transferred into a released boson from energized charged particles recoiling from electrostatic interactions. This massless boson is the force carrier for relative acceleration, which some would postulate is similar to the theorized graviton.

This energy exchange of relative acceleration would almost be similar to a Compton Scattering interaction between a photon and an electron accept this is a purely relative acceleration that is only generated through relative charged particles interacting. To be more specific, this boson can only exist through localized relative interactions for more than one particle to accelerate towards each other. This purely relative interaction results in the release of a boson that should have a more perpendicular trajectory to the plane of interaction; allowing for a plurality of generated bosons to accumulate in a single location for creating a singularity for the force of relative acceleration. Einstein's theory of General Relativity proclaimed that acceleration due to gravity is no different than acceleration due to any other force. This theory is simply reaffirming that there is no difference between gravity and acceleration, even at the atomic level, by explaining how gravity is merely the energy of relative acceleration.

Emergence may not be the best term to use, but on a grander scale it may be possible that the gradual evolution of charged particles amassing collectively in different flow patterns have allowed for the generation of gravity. For example, a charged particle without motion creates an electric field, a charged particle moving linearly creates a loop magnetic field, while a charged particle moving in a loop without curvature deviation creates a directed magnetic field. These three different states of charged particles creates different fields made of photons. If my theory of RAE in quantum mechanics is correct, curving the trajectories of charged particles relative to a single location would simply be driving a further dimension into charged particle activities that may result in the field that we know as gravity. By adding curvature to relatively interacting charged particles, relative acceleration from curvature deviation is able to influence the surrounding environment. Interesting enough a charged particle with linear, loop, or spherical motion has an electromagnetic field; and in all of our stellar observations of major gravitational phenomena an electromagnetic property is always present. For some, including Einstein, it is extremely hard to logically separate electromagnetism from gravity as other unproven theories have.

To better understand how RAE is generated amongst charged particles one needs to understand CGD. In Einstein's Theory of General Relativity [6,7] , CGD is an attractive effect between traveling objects upon a spherically shaped plane. This is a geometric effect that is depicted in the following image (a) showing two travelers that walk directly North from the Earth's Equator at the same speed from different locations. Since they are traveling on a curved surface, they will eventfully meet each other. The closing distance in-between these two travelers is the relative acceleration between them. This deviation from a directly northern path on a curved surface is a very basic example of CGD. Since CGD effects need to occur on a curved surface; all of the confining magnetic coils not on the axis of rotation on the NESAR need to be angled to create a Curved CGD Plane. The following image (b) depicts this created plane that is formed by electrons being pushed in a curved trajectory against the created photon layer of the confining magnetic fields. 

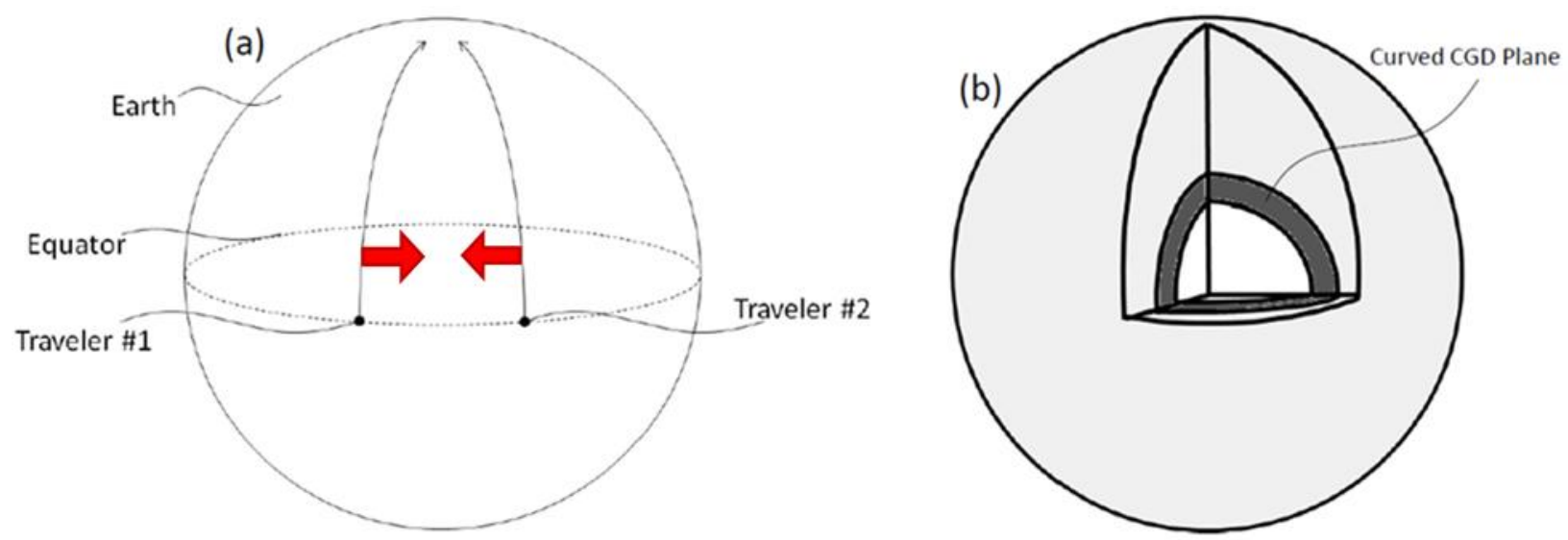

Once the confinement is set appropriately the charged particles will rotate relative to the SRCP. Electrons are preferable to confine for RAE, as they are the least massive and easiest charged particle to confine. Electrons can generate a magnitude of RAE equal of that of the proton at 1/1836 the mass. So, to create the most efficient closed system for relative acceleration energy, it must be a confinement purely made of electrons. As confined electrons rotate in curved trajectories they converge and relatively accelerate perpendicularly relative to the collective rotational direction. As these electrons converge, they electrostatically interact with each other to repulse against one another. While this repulsion is occurring, the RAE generated amongst the electrostatically interacting electrons causes them to recoil and expel scattered energy in the form of a massless boson. This generated boson is the force carrier of relative acceleration. The below diagram is a simplified depiction of this converging interaction.

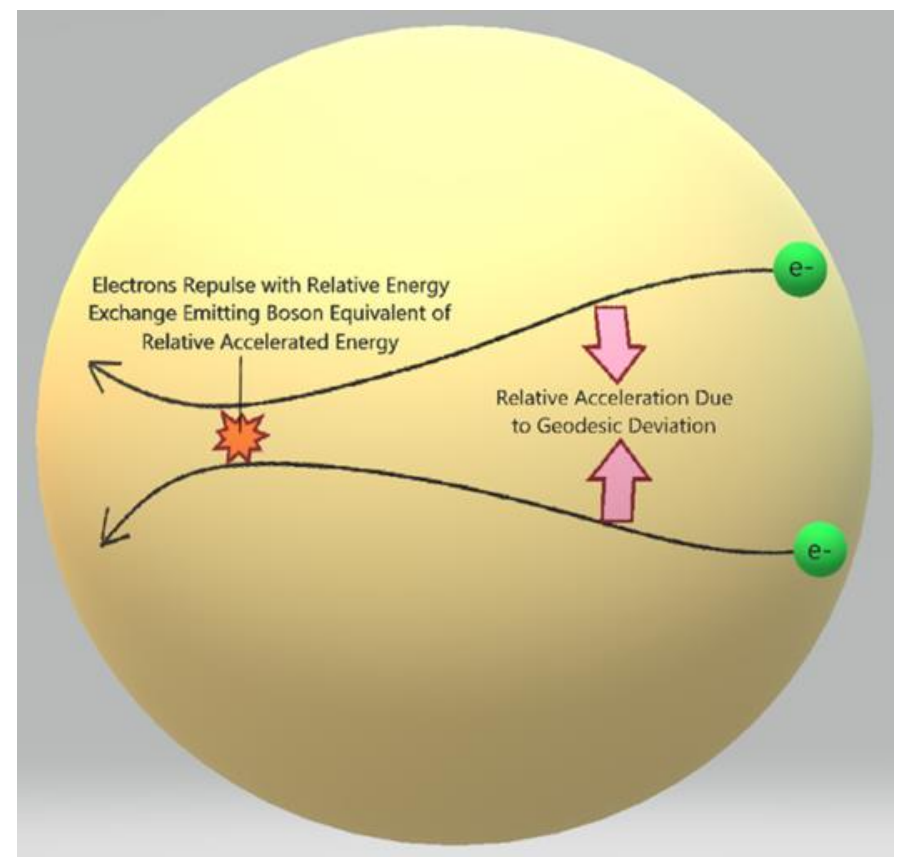

To better understand how relative RAE may have the potential to recoil amongst electrostatically interacting electrons under curved trajectories, it would be simpler to relate this interaction to a photon being absorbed by an electron. When a photon is absorbed by an electron, the 
electron's energy level increases. This increase in energy often means that the electron will increase in its momentum; but at the same time will recoil to generate another photon with less momentum. The two main differences between a photon and the energy generated from relative acceleration interacting with an electron is that relative acceleration does not exist until curvature allows objects to accelerate relatively; and that the electrostatic interaction is transferring energy between fields instead of a wave.

When a free electron accelerates linearly; electromagnetic radiation, a photon, is emitted. With that being stated, the more subtle energy of deviation creating acceleration between relative electrostatic interacting charged particles, in this case electrons, should also produce some type of boson that embodies this subtle dimension of acceleration. If the product of this relative interaction is a boson that generates RAE, then the effect of time dilation makes perfect sense. If gravity is truly a product of acceleration at the quantum level, then time would be affected simply because acceleration is completely reliant on time and relative positioning. In analyzing the behavior of time dilation, it mimics exactly how relative acceleration occurs by converging prior to repulsing; producing a product that reflects the effects of acceleration by reducing the amount of time needed for an interaction to occur. This causes a closed system's time to reduce as well by slowing time in the influence of its field. The relative nature of this interaction makes sense why time slows in environments that have stronger gravitational fields, which is essentially a field region where increased RAE occurs.

In NESAR like confinements like stars, there possibly are two types of relative interactions occurring. First possible method, which is depicted in the below diagram, would be by electrons interacting relative with the SRCP. The confined electrons in this diagram are rotating clockwise and are continuously electrostatically interacting with each other, but once an electron experiences a curved trajectory that RAE is electrostatically transferred to the other electrons. In the diagram, the electrons experiencing curved trajectories are highlighted with a yellow ring. The yellow ring's thickness represents the magnitude of RAE transferred through electrostatic interactions with the surrounding electrons.

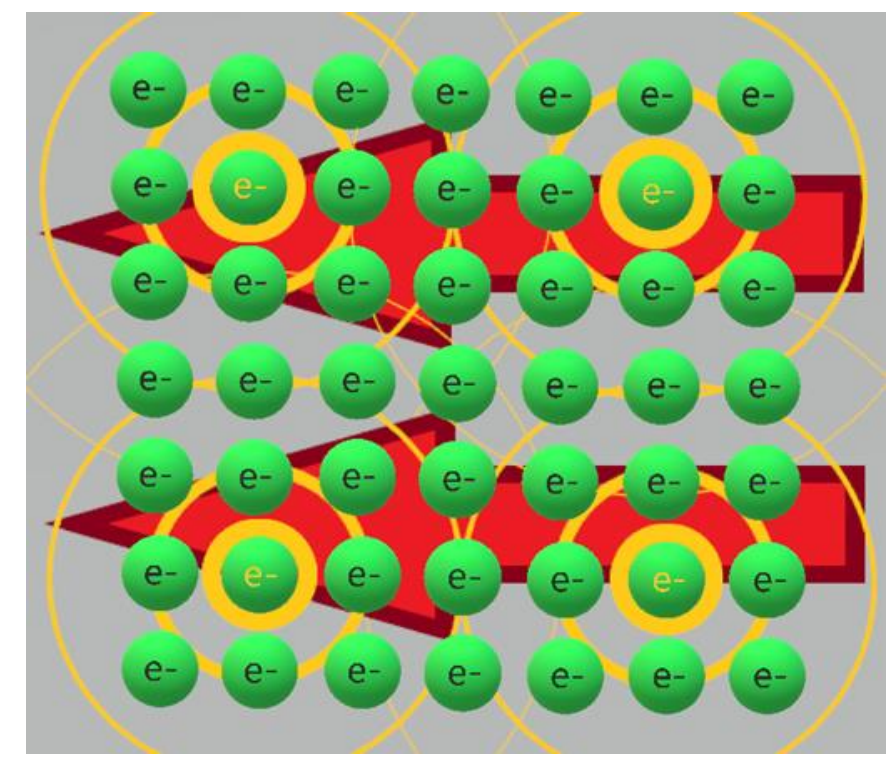

The second method, which is depicted in the below diagram, would be by electrons interacting relative with each other. The confined electrons in this diagram are rotating clockwise and are 
continuously electrostatically interacting with each other, but once an electron experiences a converging curved trajectory relative to at least one other electron experiencing the same converging curved effects. Instantaneously there is a generated RAE between the relative interacting electrons; generating a massless boson from the electrons recoiling from the energy of the relative acceleration. Again, this generated boson is the force carrier of relative acceleration. In the diagram, the electrons experiencing relative acceleration are highlighted with a yellow ring. Do note that the electrons do not need to be beside each other for relative convergence to create a relative acceleration between the two charged particles. As long as there is relative acceleration between electrons due to a curved trajectory while they are electrostatically interacting, then there is a capability to recoil and convert the generated energy from relative acceleration to a boson. In the below depiction, the generated boson due to relative acceleration energy is depicted with a purple capsule in-between the electrons.

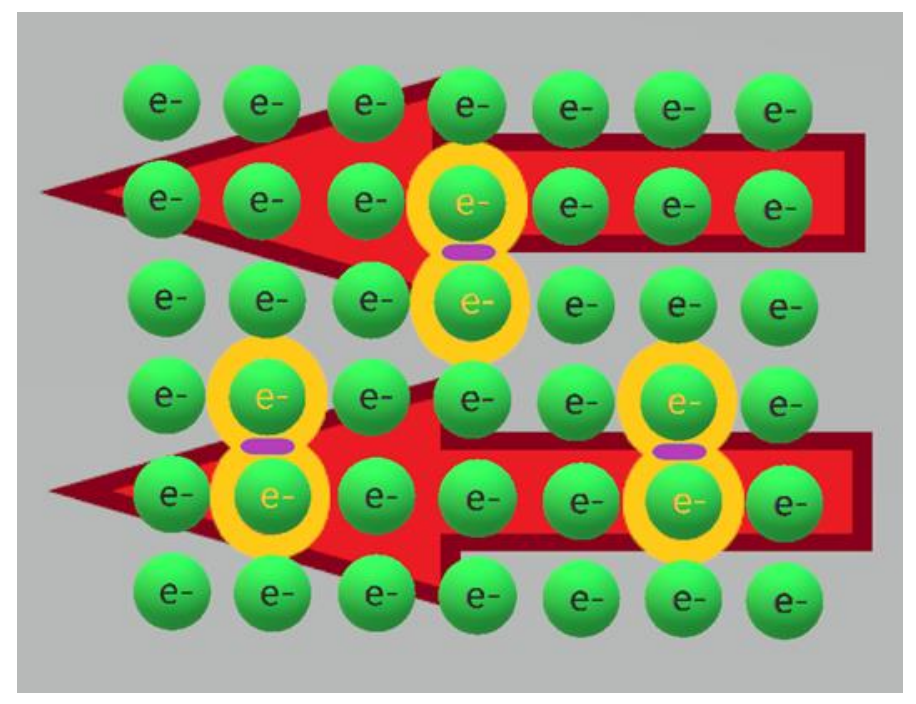

There is a high probability that both types of relative acceleration previously mentioned could be occurring simultaneously between individual electrons deviating relative to the SRCP and grouped electrons deviating relative to each other in a given closed system. To maximize the RAE in a given closed system; there are nine major influences that factor into the magnitude of the generated energy:

1) Magnetic strength of the confinement system

2) Composition ratio. Electrons/Protons ratio. The greater the ratio of electrons confined; the greater energy generated from relatively accelerated interactions at lower masses.

3) Curvature/deviation rate. The number of curvatures/deviations placed on charged particles over a given amount of time. Closed system's rotational speed and average magnitude of curvature/deviation.

4) Confinement diameter

5) System's rotational speed. The rotational speed of the charged particles, faster speeds yield more curvature events.

6) Well defined Single Relative Center Point (SRCP) defined. The more congregate the SRCP is to the collective of charged particles, a greater yield of curved trajectories will occur. 
7) The average magnitude of curvature/deviation placed upon charged particles.

8) Confinement density

9) Depth of charged particle layer on CGD plane. Testing of protype will be needed to develop a method to estimate

Gravity is a very weak force, and if the energy of relative acceleration is quantized as previously described; then there is a simple reason that the force is observed as weak. The momentum created by curved deviation is much more subtle and considerably less dynamic in comparison to electrostatic interactions that occur within a closed system, like an atom. So, it may be impossible to observe RAE at the quantum level when the composition ratio amongst almost all baryonic matter is one electron for every proton. Possibly the greatest benefit of the NESAR confinement method is its potential to create a closed system that could exponentially enhance the energy generated for relative acceleration at a fraction of the mass. This would allow for the so-called gravitational force to be measured at the atomic level for the first time.

If gravity is generated by curved trajectories upon electrostatically interacting charged particles, then this concept is able to be applied in explaining how the force of gravity is not tied to mass. Before the development of quantum mechanics, it makes sense to blindly assume that all mass obtains the gravitational force based upon observation; but with our modern knowledge of quantum mechanics, we should not settle for this simple jointed relationship. All atoms obtain at least one proton, electron, and neutron that are in constant curved trajectories about a SRCP. If atoms are operating in this manner, the electron and protons are in continual electrostatic interaction in a way that allows for the possibility to generate RAE due to curvature deviation. Without this type of interaction, the energy of relative acceleration could not be transferred into a boson; possibly ceasing the potential for RAE, gravity, to even exist. Based on this logic, there is also the potential that this accelerated energy may be held within individual protons and neutrons, which consist of up and down quarks interacting in a three-body closed system. With further research, I am hoping that this concept can assist in better understanding the strong CP problem that has led to theorizing the existence of axions. This testable theory has the potential to prove that the force of gravity is not tied to mass, RAE is purely tied to closed systems obtaining relative interacting charged particles with curved trajectories obtaining deviation. Even if my notion about all mass not having gravity is wrong, the NESAR concept still operates appropriately to understand gravity because it allows for the capability to calculate the force of gravity at a singularity.

The theory of General Relativity [6] allows for the postulation of a singularity, while the existence of a singularity goes against the laws of quantum mechanics as solutions lead to an infinite curvature when calculating gravity in extreme situations. The NESAR confinement method provides a quantifiable pathway to developing a solution that can calculate RAE generated from a closed system by factoring a given confinement's composition ratio, curvature/deviation rate, confinement density, depth of charged particle layer on CGD plane, and confinement diameter against a closed system's total mass energy. Main reason that this concept allows for a non-infinite answer at the singularity, is because the energy generated for relative acceleration never occurs at a singularity. The deviation upon charged particles interacting about the singularity generates the gravitational force for the whole system. To simplify this logic, no matter how complicated Einstein's equation is on gravitational theory; it extends from Newton's law of gravity which is essentially the gravitational constant and mass divided by the radius $\left(G M / r^{\wedge} 2\right)$. Einstein's equation, just like Newton's, yields an infinite solution when the radius is 
zero, which is not the case for the NESAR model. To simply put it, if gravitational energy is a product of relative curvature deviation; then it is impossible to originate relative curvature at a singularity where the relative distance would be zero. To calculate the gravitational energy of a closed system, one must focus on the accelerated deviations that occur between charged particles within a core's Curved CGD Plane.

In analyzing the energy of relative acceleration in this manner, it easy to see that creating a closed system that consists mainly of electrons greatly effects a closed system by greatly increasing the system's composition ratio while simultaneously exponentially reducing the mass energy of the closed system. In doing so, the generated RAE from a closed system that consists mainly of electrons has the potential to supersede the system's total mass energy; which would produce a closed system that would present similar properties that are observed with black holes. Below is a simple diagram presenting the factors that are needed in calculating a closed system's RAE, depicted in green, against a closed system's mass energy, depicted in red, to exceed the baryonic limit to create a black hole.
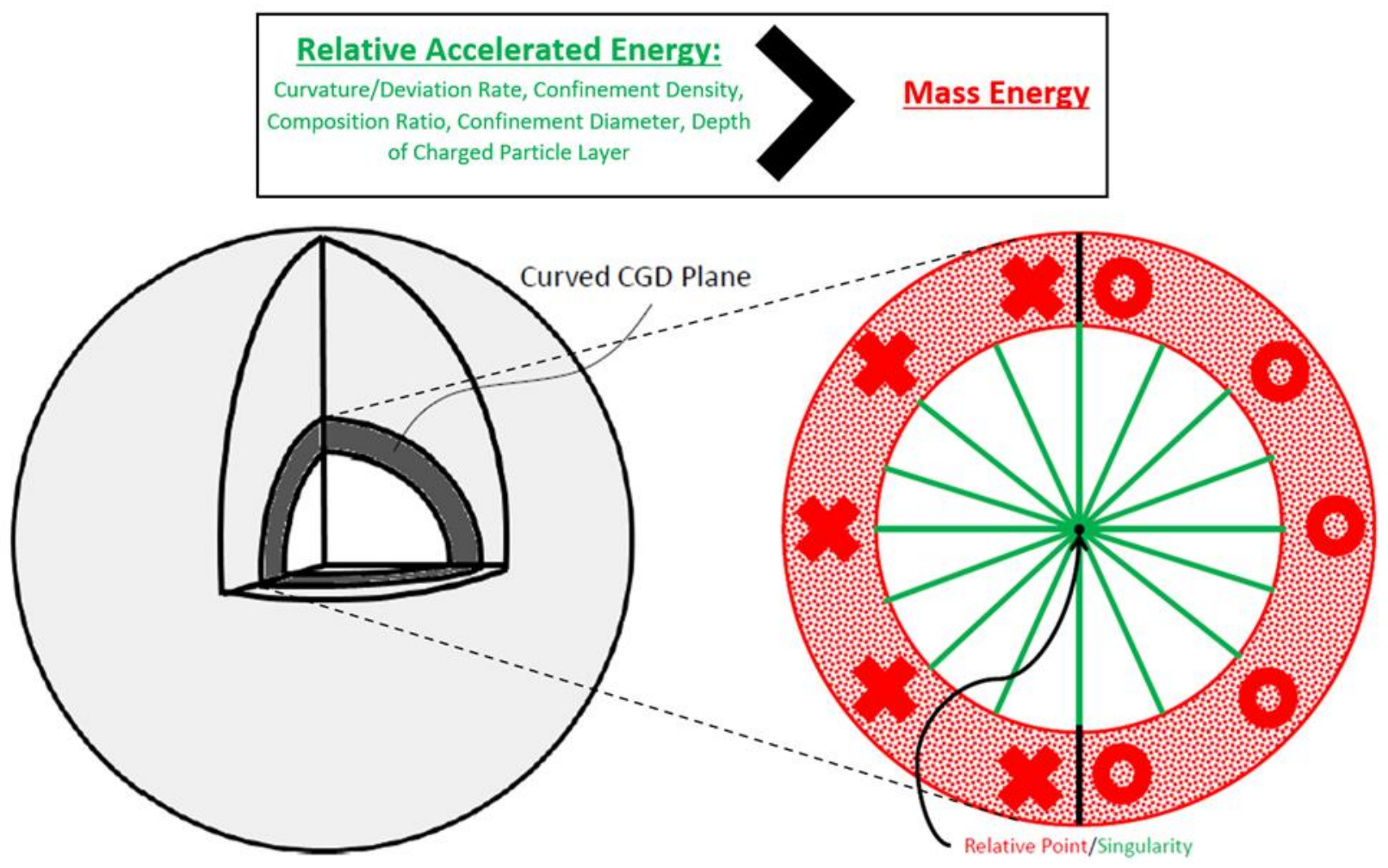

This means if a closed system is efficient enough in creating energy from curvature with the right ratio of charged particles with deviation, it has the potential to generate enough RAE to overcome the energy that is generated from a source that is limited by the mass energy of baryonic matter. A photon being generated from a hydrogen atom is an example of energy that has a baryonic limitation. When a closed system is able to supersede universal baryonic limits, it has the capability to demonstrate similar effects that are observed with dark matter. A confinement that is predominantly electrons, with some baryonic charged particles, still has the potential to generate enough RAE to surpass the systems mass energy. Even though a system like this surpasses the baryonic limits with the electrons confined, it still has a secondary RAE from baryonic matter; possibly allowing for two separate gravitational systems working within a single system. This type of observation has been observed from some black holes and 
has been deemed as evidence of dark matter. On a cosmological scale, the effects of theorized dark matter are only observed around black holes. Which means that the influences of RAE around confinements with higher composition ratios are being wrongly categorized as dark matter. Since the theory of dark matter is based upon the limits of baryonic matter, the NESAR concept has the potential to fully discredit the theory of dark matter altogether; making the Universe a little less enigmatic.

The main reason that further developing calculations based upon the NESAR confinement model have a high probability in satisfying both general relativity and quantum mechanics; is that this is the first concept that logically separates mass from the force of gravity being generated from a singularity. The reason that Einstein's equations don't work at a singularity, are because they are the wrong approach to fully understanding gravity at the quantum level. The main reason for this slight misunderstanding in the gravitational force is because baryonic mass does not play as much as a factor in generating the force of gravity in the most extreme environments like a black holes. Again, If gravity is truly a product of relative curvature/deviation, then it is simply impossible for the energy of curvature/deviation to originate from the SRCP/singularity. Energy that is relative in nature, must originate from a relative location.

I know that a greater majority of those who review this paper will have the initial instinct to oppose the logic in this theory simply because so much time, money, research, and even faith has been placed in trying to find and understand the hypothesized particle called dark matter. If the most prideful do choose to oppose this theory without logical reason, they should remember this theory came with a way to test the validity. So, if my theory is wrong, researchers will not spend countless amounts of man hours and money on something that may never be true in the first place. But, If this theory is right a whole new field of science, called Charged Particle Shaping (CPS), is born. CPS may be the needed approach to maximizing man's utilization of fusion energy and propulsion; allowing a pathway to fulfil an ever-growing population's need for energy and natural resources.

\section{How the NESAR Can Be Used for Propulsion (Anit-Gravity)}

For those who are familiar with the Riemann Curvature Tensor [7] , which is the math used to for geodesic deviation, it is known that relative moving objects on a curved surface can diverge as well converge if the surface curvature allows for deviation. In this part of the paper I will explain how the NESAR can be altered so that the generated energy of relative acceleration can be used for repulsion instead of attraction. Before getting to how to alter the NESAR to generate deviating RAE, I would like to cover where we have already observed repulsive gravitational energy in nature.

The main issue with Einstein's construct of gravity is that mass and energy originate from a singularity, when in actuality the relative interactions about a singularity are the true source of RAE for a closed system. Because of this slight error in applying relative energy, there was also an oversight in the predictions on the source of gravitational waves. What we have been observing as gravitational waves is actually a repulsive energy originating from in between two approaching gravity systems.

Depicted below are two gravitational closed systems approaching one another, there is a relative region in between the two interacting systems that is experiencing a repulsive energy. As these two sources get closer, the potential repulsive energy at the relative location in between the two systems increases. The relative region of potential repulsive energy is not a closed system, so the potential repulsive energy can't be utilized until the relative position almost develops into an SRCP. It is 
not until moments before these two systems fully merge that the repulsive energy is maximized, nearly obtaining the kinetic energy of both systems to finally generate a massive release of repulsive energy that opposes and disrupts the surrounding normal flow of spacetime known as a gravitational wave. Once this repulsive energy is released; the two systems merge into a single entity that is gravitationally attractive. In the depiction below, the growing potential repulsive energy is depicted in red; demonstrating how repulsive energy increases and maximizes in magnitude just before the two systems combine.

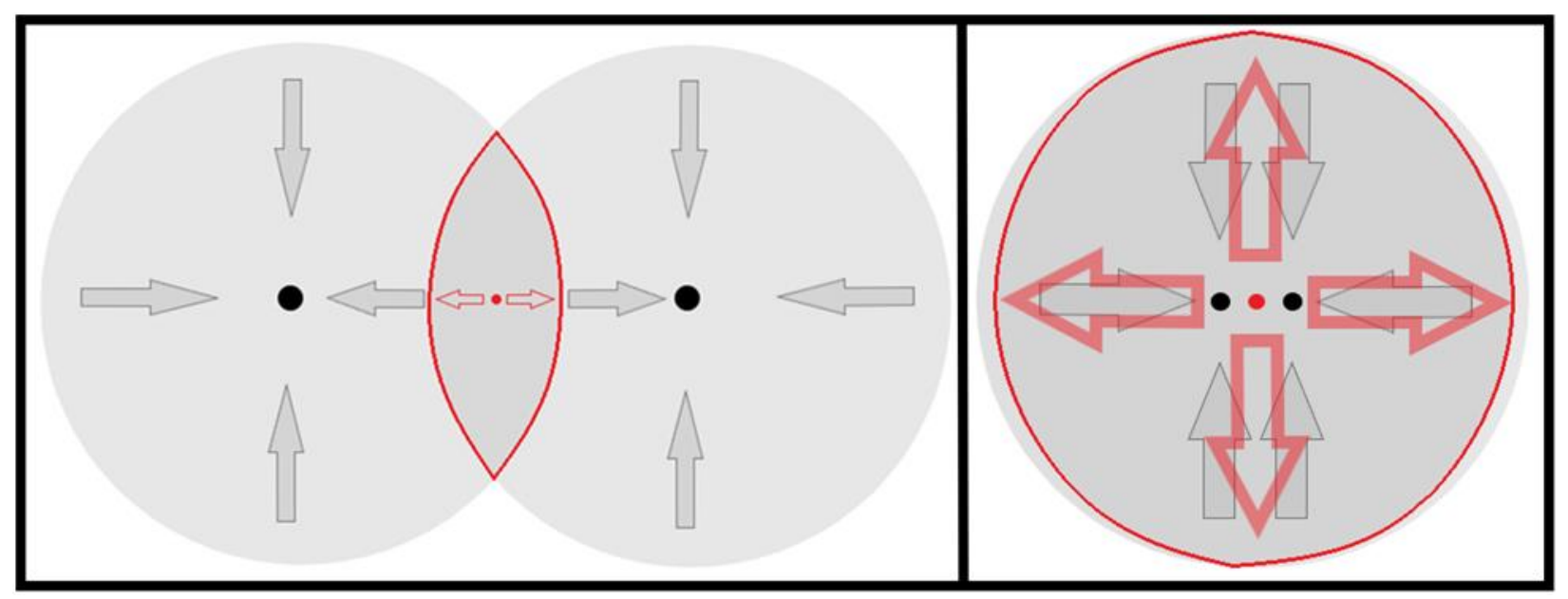

The previous depiction of how repulsive energy is generated as a gravitational wave is different from diverging RAE. I presented the logic in how this repulsive energy is generated to reiterate how important relative location is to utilize accelerated energy, by emphasizing the establishment of a location that can operate similar to a SRCP; even if it is temporary.

As for the NESAR there are two separate ways to utilize the confinement system to generate the repulsive energy known as anti-gravity. The first method would be by producing areas of confinement where curvature can influence charged particles to diverge, and the second method would be by generating repulsive energy from confining anti-matter, preferably positrons.

The first method, of producing areas of divergence upon the confines, is simply by increasing the magnetic strength of certain confinement coils in the areas where one would like to create diverging energy. In the following depiction, there is a confinement of charged particles rotating clockwise. To place a repulsive force on the top and bottom areas of the confinement; the top and bottom conductive coils of the confinement apparatus have been increased in strength to push a diverging curvature on the confines. In doing so, diverging energy is generated at the top and bottom areas of the confines. 


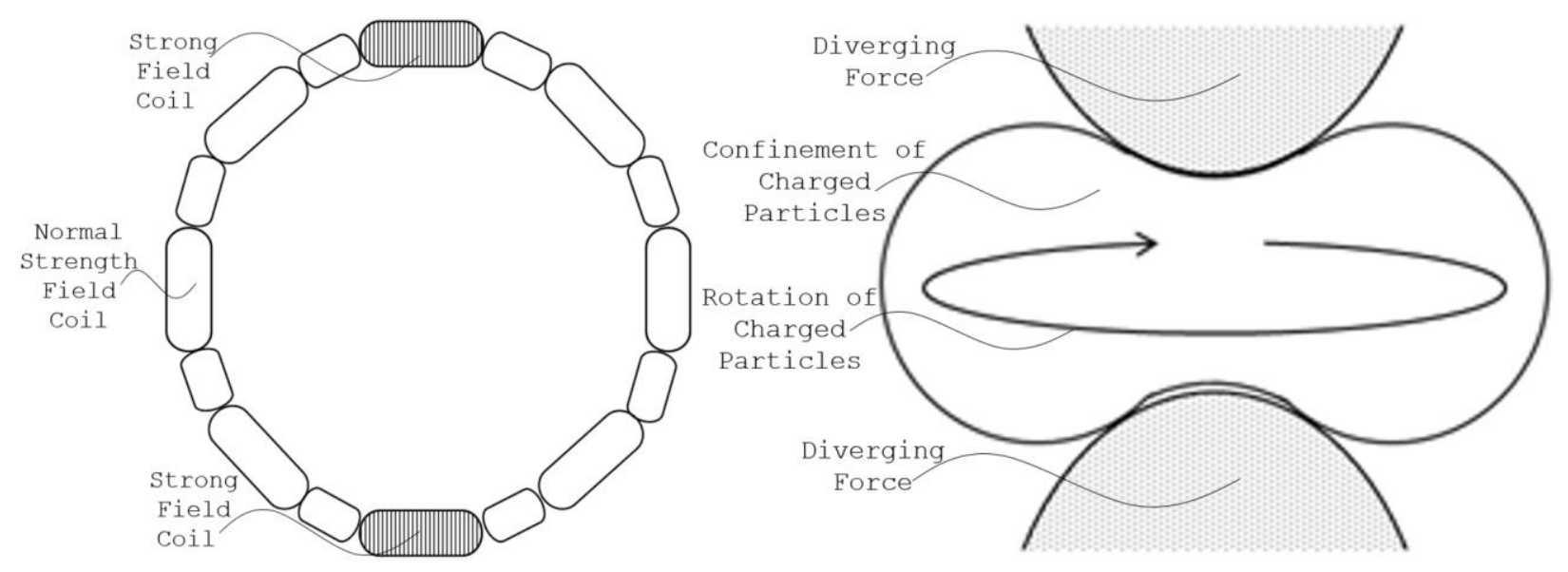

The Second method, of producing a repulsive force would be by using the NESAR in confining positrons instead of electrons. A confinement of positrons should confine exactly like electrons; so I am somewhat skeptical that this approach may yield a repulsive energy. If by some chance a confinement of positrons are able to produce repulsive energy then there is a great probability that this type of confinement can show how dark energy is generated; and finally providing an answer on where has all of the anti-matter may have gone in our universe.

\section{Conclusion}

This is an unconventional theory with an unconventional product. Unlike other quantum theories of gravity that have produced calculations stemming from unvalidated postulations with little to no way of testing the validity of the claims; this theory's product is the complete opposite stemming from verified deviations and laws with a device to test the validity of the postulations. Once a prototype can be built and tested to validate this theory, it should be emphasized that developing a possible lawful quantum theory of gravity was not an issue of developing new math to solve the problem, the issue was finding a way to understand the problem from a different perspective.

Rearranging the scientific method of approach may have contributed to being able to obtain a different perspective by developing an experiment from observations before constructing any type of hypothesis on gravity or fusion. To better understand how the sun operates, I designed something that could possibly function similarly to it. The logic in this approach was that a better understanding of the sun could be achieved through analysis and experimentation on a device that works like it. In the normal scientific approach, it is standard to develop the theoretical prior to the experimental. So, it may be due to an unorthodox scientific approach that assisted in providing the needed perspective in developing this theory of quantum gravity.

To date I have developed this theory in solitude with only God's guidance. I am at a precipice of my limitations with this novel concept and have gone as far as I can by myself. Now is the time to collaborate for further progression on this testable theory. The next step is to conduct 3D simulations, shortly followed by building a prototype for testing. If this theory is correct, it should not be hard to experiment on a confinement consisting only of electrons to generate possible RAE. If experiments yield positive results in confirming that this theory is correct, numerous following experiments need to be 
conducted with different composition ratios to start refining measurements to develop an allencompassing equation of quantum gravity.

The application based upon the NESAR has the potential to exponentially advance man's capabilities of sustainable fusion and propulsion to almost boundless limits. Finally, allowing for constant acceleration in space propulsion that could provide interplanetary travel in a matter of hours instead of months and years. This concept also extends man's capabilities in obtaining unlimited natural resources beyond Earth; which may become necessary for a vastly growing population. Ignoring the possible capabilities of NESAR is irrational when compared to the potential gains. To those who understand that this could be the beginning of a new frontier in scientific advancement and discovery; this theory should be rousing and exciting in comparison to other theories that have no application capabilities.

\section{$\underline{\text { References }}$}

[1] Moss, Samuel (2019) Fusion Energy Device With Geodesic Deviation Gravitational Effects. U.S. Patent Application No. 20190088375(A1). Washington, DC: U.S. Patent and Trademark Office.

[2] Ohanian, Hans (1976). Gravitation and Spacetime (1st ed.).

[3] Nave, Carl. (n.d.). Hyperphysics. Retrieved November 22, 2021, from http://hyperphysics.phyastr.gsu.edu/hphys.html.

[4] Igochine, Valentin. Recent Progress in MHD Simulations and Open Questions. Aug. 2017, https://www.slideserve.com/ophrah/recent-progress-in-mhd-simulations-and-open-questions.

[5] Zell, Holly. (2017, Aug. 7). Themis - Search Coil Magnetometer (SCM). NASA.

https://www.nasa.gov/mission_pages/themis/spacecraft/SCM.html.

[6] Einstein, Albert. Die Grundlage der allgemeinen Relativitatstheorie. Annalen der Physik, 354:769-822, 1916.

[7] Carroll, Sean (2004). Spacetime and Geometry. 